\title{
A importância da prática de mindfulness como ferramenta para a redução dos sintomas de ansiedade e depressão no contexto da pandemia de covid-19
}

The importance of mindfulness practice as a tool to reduce anxiety and depression symptoms in the context of the covid-19 pandemic

La importancia de la práctica del mindfulness como herramienta para reducir los síntomas de ansiedad y depresión en el contexto de la pandemia del covid-19

\section{Resumo}

Com sua origem nas práticas budistas, mindfulness tem como objetivo desenvolver a consciência do que estamos pensando, sentindo e fazendo no momento presente. A prática de mindfulness vem ganhando espaço na psicologia ocidental desde os anos de 1700 , e desde então sua eficácia com relação à promoção de saúde mental e remissão dos sintomas de transtornos mentais, sendo comprovada através de pesquisas científicas. Diante disso, se faz necessário realizar um paralelo com a pandemia do covid-19, que está causando impactos nos aspectos psicológicos dos indivíduos, levando alguns a desenvolverem sintomas de ansiedade e depressão. Nesse sentido, o objetivo deste trabalho é analisar a importância da prática de mindfulness como ferramenta para a redução dos sintomas de ansiedade e depressão no contexto da pandemia do covid-19. Por isso, o presente trabalho propõe realizar um levantamento bibliográfico através das plataformas Google Acadêmico, SciELO e PubMed, categorizando os capítulos de livros e artigos para a análise dos resultados a partir de: a) mindfulness como intervenção para a diminuição dos sintomas de ansiedade, depressão e outros transtornos mentais e; b) mindfulness como intervenção para a diminuição dos sintomas de estresse causado na população em virtude da pandemia de covid-19. Sendo assim, os resultados obtidos a partir dessa revisão bibliográfica apontam que a intervenção em mindfulness é eficaz e eficiente enquanto estratégia de enfrentamento ao sofrimento psicoemocional decorrente da pandemia do covid-19, estando associado a prevenção, promoção e tratamento da saúde mental.

Palavras-chave: Ansiedade; Covid-19; Depressão; Mindfulness.

\begin{abstract}
With its origins in buddhist practices, mindfulness aims to develop an awareness of what we are thinking, feeling, and doing in the present moment. The mindfulness practice has been gaining ground in western psychology since the $1700 \mathrm{~s}$, after that, its effectiveness in promoting mental health and remitting symptoms of mental disorders has been proven through scientific research. Therefore, it is necessary to draw a parallel with the covid-19 pandemic, which is impacting the psychological aspects of individuals, leading some to develop symptoms of anxiety and depression. Then, this study aims to analyze the importance of mindfulness practice as a tool to reduce anxiety and depression symptoms in the context of the covid-19 pandemic. Hence, the present work proposes to carry out a bibliographic survey through the Academic Google, SciELO, and PubMed platforms, categorizing the chapters of books and articles to analyze the results: a) mindfulness as an intervention to reduce symptoms of anxiety and depression and other mental disorders and; b) mindfulness as an intervention to reduce the stress symptoms caused in the population due to the covid-19 pandemic. Therefore, the results obtained in this bibliographic review points that mindfulness intervention is effective and efficient as a coping strategy for psycho-emotional suffering resulting from the covid-19 pandemic, being associated with prevention, promotion, and treatment of mental health.
\end{abstract}

Keywords: Anxiety; Covid-19; Depression; Mindfulness. 


\begin{abstract}
Resumen
Con sus orígenes en las prácticas budistas, la atención plena tiene como objetivo desarrollar la conciencia de lo que estamos pensando, sintiendo y haciendo en el momento presente. La práctica de la atención plena ha ido ganando terreno en la psicología occidental desde el siglo XVIII y, desde entonces, su eficacia para promover la salud mental y la remisión de los síntomas de los trastornos mentales ha sido probada a través de la investigación científica. Por lo tanto, es necesario establecer un paralelo con la pandemia del covid-19, que está impactando los aspectos psicológicos de los individuos, llevando a algunos a desarrollar síntomas de ansiedad y depresión. En este sentido, el objetivo de este estudio es analizar la importancia de la práctica del mindfulness como herramienta para reducir los síntomas de ansiedad y depresión en el contexto de la pandemia del covid-19. Por ello, el presente trabajo propone realizar un relevamiento bibliográfico a través de las plataformas Academic Google, SciELO y PubMed, categorizando los capítulos de libros y artículos para el análisis de los resultados de: a) Mindfulness como intervención para reducir los síntomas de ansiedad, depresión y otros trastornos mentales y; b) Mindfulness como intervención para reducir los síntomas de estrés provocados en la población por la pandemia del covid-19. Así, los resultados obtenidos de esta revisión de la literatura indican que la intervención de mindfulness es eficaz y eficiente como estrategia de afrontamiento del sufrimiento psicoemocional derivado de la pandemia del covid-19, estando asociada a la prevención, promoción y tratamiento de la salud mental.
\end{abstract}

Palabras clave: Ansiedad; Covid-19; Depresión; Mindfulness.

\title{
1. Introdução
}

Mindfulness, também conhecido como atenção plena, é uma prática que pode ser desenvolvida e treinada com o objetivo de desenvolver a consciência do que estamos pensando, sentindo e fazendo no exato momento, é prestar atenção no momento presente, no aqui e agora. De acordo com Jon Kabat-Zinn (1990), mindfulness é uma consciência sem julgamentos, momento a momento, cultivada por prestar atenção de maneira específica, isto é, no momento presente, de forma não reativa e com o coração mais aberto. A atenção plena traz benefícios à saúde mental, pois há um reconhecimento dos pensamentos, emoções e interpretação das vivências.

Mindfulness tem sua origem nas práticas budista, há mais de dois mil e quinhentos anos, mas foi apenas no final dos anos de 1700 que as práticas orientais começaram a ser estudadas no ocidente. Teóricos como Sigmund Freud, Franz Alexander e Carl Jung demonstraram interesse nas psicologias orientais. Ainda, nas décadas de 1960 e 1970 muitos estudiosos, entre eles terapeutas, se dedicaram a estudar sobre mindfulness. Entre esses estudiosos, estava o psicoterapeuta e psiquiatra Fritz Perls, que estudou o zen budismo e mais tarde introduziu o mindfulness na prática psicoterapêutica da Gestalt-terapia. Em 1979, o médico Jon Kabat-Zinn fundou o Programa de Redução do Estresse baseado em Mindfulness (MBSR) na Universidade de Massachusetts nos Estados Unidos, se tornando o principal programa sobre mindfulness utilizado em pesquisa psicológica (Germer, Siegel, \& Fulton, 2016). Dessa forma, aos poucos, as práticas da psicologia budista foi encontrando espaço na psicologia ocidental, e diversas pesquisas foram sendo realizadas para comprovar a eficácia do uso de mindfulness no tratamento de transtornos mentais.

Sobre isso, El Morr, Ritvo, Ahmad, Moineddin e Equipe (2020) desenvolveram um estudo de intervenção de 8 semanas, com práticas de atenção plena online em 160 alunos, a fim de diminuir os níveis de sintomas de depressão, estresse e ansiedade, através do uso de conteúdos de psicoeducação de prática de mindfulness, fóruns de discussão e práticas online com instrutores. Os resultados nos escores de ansiedade, estresse e depressão foram colhidos através de instrumentos como o Inventário de Ansiedade de Beck (BAI), Formulário Curto do Questionário de Mindfulness das Cinco Facetas (FFMQ-SF), entre outros instrumentos. Ao final das 8 semanas, foram realizadas novas coletas de resultados, demonstrando uma redução significativa nos níveis de ansiedade e depressão dos participantes e aumento nos escores de atenção plena. Logo, esse estudo evidenciou a eficácia das práticas de atenção plena em indivíduos com demandas de ansiedade e depressão.

Levando em consideração os aspectos apresentados e a importância do uso de mindfulness como ferramenta para a promoção de saúde mental, se faz necessário estabelecer um paralelo com o contexto da crise mundial de saúde, onde a Organização Mundial de Saúde (OMS), em março de 2020, declarou o estado de pandemia do Coronavírus Disease 2019 
(COVID-19), adotando inúmeras medidas para conter o contágio da doença, sendo o distanciamento social uma das mais importantes e urgentes a ser implementada (Bezerra, Silva, Soares, \& Silva, 2020). Contudo, a estratégia de isolamento social e as medidas para conter o avanço da transmissão do covid-19 vêm causando inúmeros impactos na sociedade, ocasionando mudanças econômicas e sociais, alterando não só o comportamento das pessoas, como também acarretando em impactos nos aspectos psicológicos da população. Nesse sentido, em um estudo transversal realizado por Barros, et al., (2020) demonstra que em uma amostra com 45.161 brasileiros, incluindo indivíduos de todos os estados do país, $40 \%$ dos participantes adultos apresentaram emoção de tristeza, enquanto 50\% dos indivíduos apresentaram ansiedade diante do contexto da pandemia do covid-19.

Portanto, com a pandemia do covid-19, a saúde mental vem ganhando espaço nos debates da sociedade e embora ainda exista inúmeros estereótipos que circundam a prática profissional em psicologia, com o avanço e a acessibilidade das informações, principalmente através das comunidades virtuais, os profissionais psicólogos passam a usar as redes sociais para disseminar informação, levando acesso ao conhecimento técnico-cientifico da psicologia para a população e contribuindo para a prevenção de transtornos mentais. Durante a pandemia do covid-19, notou-se um movimento nas comunidades virtuais de maior abertura e incentivo aos tratamentos e intervenções que visa a saúde mental da população, principalmente se tratando de demandas de transtornos depressivos e ansiosos.

Dados da Organização Mundial de Saúde (OMS) (2017) apontam que o transtorno mental mais recorrente no mundo é a depressão, acarretando mais de 300 milhões de pessoas, em sua maioria, mulheres. Segundo o Manual Diagnóstico e Estatístico de Transtornos Mentais, $5^{\text {a }}$ edição, (DSM-5) o transtorno depressivo maior, por exemplo, pode ser caracterizado pela presença de cinco ou demais sintomas diagnósticos, estando presente os sintomas de humor deprimido, avolia e/ou anedonia em um período de pelo menos duas semanas (American Psychiatric Association, 2014).

Em relação aos transtornos ansiosos, o DSM-5 caracteriza o transtorno de ansiedade generalizada como a presença de ansiedade e preocupação excessiva referente à diversas situações, que podem apresentar risco real ou imaginário ao paciente, presentes de maneira intensa e duradoura em um período de pelo menos seis meses (American Psychiatric Association, 2014). Segundo o Ministério da Saúde (2020), durante o período de pandemia do covid-19, o transtorno de ansiedade se tornou o mais frequente na população brasileira. Ainda, verificou-se a presença de elevados níveis de transtorno de estresse pós-traumático e transtorno depressivo. A pesquisa demonstrou que a maioria dos afetados são mulheres, corroborando com os dados da Organização Mundial de Saúde apresentados acima.

Ou seja, o distanciamento social somado ao período de quarentena levara as pessoas a apresentarem sintomas de ansiedade e até mesmo quadros depressivos, assim como outras demandas psicoemocionais que afetam a saúde mental da população.

Dessa forma, devido a sua importância no contexto atual, o presente artigo tem como propósito analisar e divulgar o conhecimento e a importância das técnicas de mindfulness, bem como, seus impactos na qualidade de vida das pessoas, devido ao grande aumento dos níveis de sintomas de ansiedade e depressão decorrente da pandemia do covid-19, sendo a prática de atenção plena uma estratégia de enfrentamento de extrema importância na busca por saúde mental.

\section{Metodologia}

Neste trabalho foi realizado o levantamento bibliográfico através das plataformas Google Acadêmico, SciELO e PubMed com publicações entre os anos 2015 e 2021. Nas pesquisas foram utilizadas as palavras-chave "mindfulness", "covid 19", "ansiedade" e "depressão". Foram inclusos nesse estudo artigos originais publicados no Brasil e em revistas nacionais, artigos de língua inglesa e capítulos de livros. 
Na plataforma Google Acadêmico, ao realizar as pesquisas utilizando as palavras-chave "mindfulness" acrescentando com and "covid 19", entre os anos 2019 e 2021, foram encontrados 8.850 trabalhos com esses descritores. Quando pesquisado as palavras-chave "mindfulness" acrescentando com and a palavra "ansiedade" e or "depressão", entre os anos 2015 e 2021, foram encontrados 3.300 resultados. Foram selecionados 45 artigos, após a leitura detalhada dos resumos foram excluídos 40 por não contemplar os objetivos do trabalho, restando 05 artigos da plataforma Google Acadêmico.

$\mathrm{Na}$ base de dados PubMed, ao buscar trabalhos utilizando as palavras-chave "mindfulness" acrescentando com and “covid 19”, entre os anos 2019 e 2021, foram encontrados 229 artigos. Desses 229 trabalhos foram selecionados 48 para leitura dos resumos. Após análise dos resumos, 39 não condiziam com a proposta do trabalho, sobrando 09 artigos para nortear o presente trabalho.

$\mathrm{Na}$ base de dados do SciELO, ao buscar artigos com as palavras-chave "mindfulness" acrescentando com and a palavra "ansiedade" e or "depressão", entre os anos 2015 e 2021, foram encontrados 152 trabalhos. Desses 152 resultados, 42 tiveram seus resumos analisados, foram excluídos 37, restando 05 artigos a serem utilizados.

Portanto, a partir das pesquisas realizadas nas plataformas Google Acadêmico, SciELO e PubMed, foram selecionados ao todo 19 artigos originais com a finalidade de auxiliar nas pesquisas do presente estudo.

A análise dos resultados foi realizada a partir da categorização dos capítulos de livros e artigos escolhidos para compor a presente revisão bibliográfica. Sendo assim, os resultados foram categorizados a partir de: a) mindfulness como intervenção para a diminuição dos sintomas de ansiedade e depressão; b) mindfulness como intervenção na prevenção da saúde mental e estratégia de enfrentamento aos sintomas ansiogênicos e depressores ocasionados na população em virtude da pandemia de covid-19.

\section{Resultados e Discussão}

Nesta sessão de resultados e discussão, serão apresentados os princípios e fundamentos de mindfulness, bem como, seus benefícios e resultados enquanto estratégia interventiva em saúde mental e sua correlação positiva com comportamentos preventivos de saúde, contextualizando com a pandemia do covid-19. Por fim, através da apresentação de estudos de casos presentes na literatura, será descrito uma possível intervenção em mindfulness durante o processo psicoterapêutico.

\subsection{Mindfulness}

Mindfulness origina-se do termo sati, da língua pali, que pode significar um estado de atenção, de consciência no momento presente. O termo mindfulness pode ser descrito como um constructo teórico, como exercícios e práticas de meditação e, ainda, como mecanismos mentais e cerebrais que podem ser acionados e/ou desenvolvidos a partir dos exercícios de atenção plena (Germer, Siegel, \& Fulton, 2016).

Enquanto conceito, mindfulness pode ser definido como o estado em alerta momento a momento (Germer, Siegel, \& Fulton, 2016), tomar consciência e experienciar os eventos de forma clara, estando aberto para aceitar a realidade tal como se mostra (Neff \& Germer, 2019). Ou seja, o estado de mindfulness proporciona ao indivíduo uma maior conexão com as suas sensações, percepções, emoções e comportamentos. Significa desenvolver maior abertura, curiosidade e aceitação a situações cotidianas, inclusive aquelas que podem causar um estado de estresse e desconforto.

Com relação à prática de mindfulness, ela pode ser caracterizada como prática formal e informal, a prática formal diz respeito ao estado de introspecção durante um período meditativo, enquanto a prática informal refere-se a aplicabilidade da atenção plena nas pequenas ações realizadas diariamente, como estar consciente e atento a respiração, as emoções e sensações corporais, ao ambiente físico em que se encontra, enquanto realiza as refeições, escova os dentes, toma banho, caminha, entre outras atividades (Germer, Siegel, \& Fulton, 2016). 
Por fim, a prática dos exercícios de mindfulness podem ocasionar alterações cerebrais, Segundo Germer, Siegel e Fulton (2016), a prática meditativa de atenção plena pode diminuir a produção dos hormônios causadores do estresse e aumentar os biomarcadores que estão relacionados com o relaxamento físico.

\subsubsection{Mindfulness Terapêutico}

Estudos apontam que a prática de mindfulness como intervenção no processo psicoterapêutico, demonstra-se eficaz na redução dos sintomas de estresse, ansiedade e depressão (Bezerra, 2018; Almeida, Demarzo, \& Neufeld, 2020). O uso de técnicas de mindfulness também aumentam os níveis de atenção plena, resiliência e qualidade de vida (Lemos, Carvalho, Mendes, \& Brys, 2021). Na mesma perspectiva, pesquisas recentes apontam sua eficácia como intervenção adicional em adolescentes com transtornos emocionais com a presença de diagnósticos de comorbidades (Maleki, Khorramnia, Foroughi, Amiri, \& Amiri, 2021). Ainda, práticas de mindfulness com pacientes diagnosticados com transtorno de personalidade esquizotípica contribuiu para a redução da ansiedade social e a desconfiança (Antonova, Amaratunga, Wright, Ettinger, \& Kumari, 2016). Com adultos de meia-idade e idosos com sintomas depressivos, mindfulness auxiliou na diminuição da ruminação e aumento da resiliência, e, ainda, os efeitos negativos causados pelo estresse geraram menos impacto para os praticantes com maiores níveis de atenção plena (De Frias \& Whyne, 2015). Por fim, os resultados demonstram aumento na eficácia do tratamento de antidepressivos combinados com a prática de atenção plena, conforme apresenta Foroughi, et al., (2020) que realizaram um estudo com pacientes com depressão resistente ao tratamento, onde dividiu-se os participantes em um grupo experimental e um segundo grupo controle. Foi utilizado a Terapia Cognitiva Baseada em Mindfulness (MBCT) e antidepressivos no grupo experimental, enquanto o grupo controle recebeu apenas antidepressivos. Os resultados demonstraram redução nos níveis de depressão, redução da ruminação e aumento da compaixão e atenção plena no grupo experimental em comparação com o grupo controle.

Observa-se que os resultados apresentados até aqui constatam que a prática de mindfulness pode ser utilizada como medida interventiva e de ações de cuidado à saúde mental dos seus praticantes. Contudo, vale salientar que sua prática não substitui a psicoterapia e/ou a farmacoterapia no tratamento de transtornos mentais, no entanto, pode estar associada a esses tratamentos e auxiliar como resposta de enfrentamento a situações estressantes.

\subsubsection{Mindfulness e Abordagens Psicoterapêuticas}

Em psicoterapia e/ou demais modalidades de atendimentos psicológicos, mindfulness tem sido associado e incorporado a diversas teorias e abordagens psicológicas, tais como: terapia cognitivo-comportamental, psicoterapia psicodinâmica, psicoterapia humanista, psicologia positiva, entre outras. Baseado em evidências científicas, os quatro programas pioneiros de mindfulness são: Redução do Estresse Baseado em Mindfulness (MBSR) (Kabat-Zinn, 1990) no tratamento de condições crônicas, com mais de 700 programas oferecidos ao redor do mundo em 2012; Terapia Cognitiva Baseada em Mindfulness (MBCT) (Segal, Williams, \& Teasdale, 2012), que une as estratégias da psicologia cognitivocomportamental com os princípios da redução do estresse baseado em mindfulness com ênfase em pacientes com recaída de depressão; Terapia Comportamental Dialética (DBT) (Linehan, 1993) e; Terapia de Aceitação e Compromisso (ACT) (Hayes, Strosahl, \& Eilson, 1999). Outros programas de mindfulness veem se disseminando na prática em psicologia ao longo do tempo (Germer, Siegel, \& Fulton, 2016).

$\mathrm{Na}$ terapia cognitivo-comportamental, os exercícios de mindfulness buscam desenvolver um estado amplo de consciência sobre os pensamentos, emoções e sensações, esse conjunto sendo denominado como consciência metacognitiva. A metacognição busca autorregular a atenção focal, dessa forma, o objetivo primordial de mindfulness na terapia cognitivocomportamental é conduzir a mente ao estado presente (Bezerra, 2018). 
Além disso, sua utilização visa encorajar a aceitação de experiências internas, assim como expor o indivíduo a estímulos consideradas por ele aversivos, com o objetivo de conectar o paciente ao momento presente, ao mesmo tempo que promove uma reestruturação cognitivo-comportamental. Ainda, a forma como os indivíduos processam os pensamentos disfuncionais é o principal fator que acarreta recaída na depressão, portanto, a atenção plena auxilia na identificação dos sinais de instabilidade emocional para enfrentar de forma adequada os pensamentos que causam recaídas depressivas (Bezerra, 2018).

Ou seja, embora a origem da prática de atenção plena seja datada de mais de dois mil e quinhentos anos atrás, e desde então tem sido utilizada no alivio do sofrimento psicológico, é relativamente recente sua associação com a psicologia ocidental. Contudo, diante dos achados, é perceptível o quanto os benefícios e a incorporação de mindfulness vem sendo utilizado como medida interventiva em saúde mental.

\subsection{Principais Impactos da Pandemia do Covid-19 na Saúde Mental}

Diante do estado de pandemia do covid-19 e as consequentes medidas de prevenção e proteção para refrear o número de contágio decorrente do vírus, a população mundial precisou desenvolver estratégias de enfrentamento e adaptação à nova realidade que surgia de forma inesperada e, por vezes, assustadora. $\mathrm{O}$ cuidado à saúde física começou a estar presente nas pautas diárias da sociedade, e embora a infecção do coronavírus possa desencadear sintomas que geram mal estar físico, sintomas de origem psicológica e emocional também são evidenciados, como a raiva, solidão, ansiedade, entre outros aspectos psicoemocionais que podem gerar sofrimento nos indivíduos (Xiang, et al., 2020). A pandemia representa um grande desafio para o sistema de saúde mundial, no entanto, seus impactos não afetam apenas a saúde física, como também a saúde mental de milhões de pessoas (Rodriguez-Vega, et al., 2020).

Durante o último ano pandêmico, é perceptível o quanto a saúde mental da população necessita de ações de cuidado, estudos apontam que o risco de infecção em entes queridos, a busca por informações nas mídias sociais a respeito da pandemia e a ansiedade são considerados os fatores que mais desencadeiam medo na população diante no contexto da pandemia do covid-19 (Mertens, Gerritsen, Duijndam, Salemink, \& Engelhard, 2020). Na mesma perspectiva, Saricali, Satici, Satici, GocetTekin e Griffiths (2020) apresentam que a preocupação excessiva com predições futuras, bem como a incerteza, pode levar o indivíduo a desenvolver sentimentos de desesperança. Ainda, dados que geram preocupação e alerta são os casos de suicídio relatados durante a pandemia (Gonzalez-Diaz, Cano, \& Pereira-Sanchez, 2020; Bhuiyan, Sakib, Pakpour, Griffiths, \& Mamun, 2020).

A pandemia acarretou em grandes problemas e desafios para a população, e medidas tiveram que ser tomadas na tentativa de diminuir a disseminação do vírus, como por exemplo: obrigatoriedade do uso da máscara, lavar as mãos e uso do álcool em gel, distanciamento social e restrição de pessoas em locais públicos. Ou seja, todas essas medidas preventivas aumentam a proteção à população contra a propagação do vírus, contudo, adaptar-se a tal realidade pode provocar efeitos na saúde mental da população, sendo necessárias medidas de cuidados a nível psicoemocional. Nesse sentindo, Haliwa, Lee, Wilson e Shook (2020) observaram que a atenção plena pode estar associada a ações preventivas de saúde do covid-19.

Diante desse contexto, é perceptível a importância de desenvolver respostas adaptativas, funcionais e saudáveis que busque a prevenção e tratamento de transtornos mentais no período pandêmico, sendo a prática de mindfulness um possível aliado na busca por saúde mental.

\subsection{Mindfulness como Estratégia de Enfrentamento na Pandemia do Covid-19}

Os estudos sobre a intervenção baseada em mindfulness para a promoção do bem estar das pessoas durante a pandemia do covid-19 demonstram resultados positivos em relação a melhora não só da saúde mental, como também 
benefícios na saúde física desses indivíduos. Além disso, foram demonstrados que os indivíduos praticantes de mindfulness estão em contato com os próprios sentimentos, sabendo reconhecer e regular suas emoções (Rodriguez-Vega, et al., 2020).

Estudos comprovam que a melhoria na saúde mental está relacionada principalmente à diminuição de pensamentos automáticos e negativos. Nesse sentido, segundo Zhang, Zhang, Liu, Xiao e Wang (2021), os benefícios da prática de mindfulness contribui para o aumento da atenção ao momento presente, diminuindo pensamentos automáticos e negativos relacionados ao atual cenário mundial. Ainda, os autores salientam que intervenções utilizando técnicas de meditação guiada visa intervir nos processos cognitivos dos indivíduos de forma direta, aumentando a capacidade do indivíduo de se desligar de eventos negativos ou adversos, reduzindo, portanto, a ansiedade, por exemplo (Zhang, et al., 2021). A atenção plena contribui para um crescente número de benefícios dos fatores de proteção do funcionamento psicológico durante situações de alto estresse e ansiedade (Conversano, et al., 2020).

O praticante de mindfulness, quando se depara com altos níveis de sofrimento relacionados a algum tipo de estresse, tende a interpretar esse evento de uma forma mais positiva, buscando estratégias de enfrentamento adequadas a essa situação, diminuindo, portanto, a sobrecarga emocional. A atenção plena traz benefícios também em relação aos relacionamentos interpessoais, pois, os indivíduos tendem a ter uma consciência maior sobre o que podem estar lhe causando danos emocionais ou desconforto. Dessa forma, buscam regular as emoções por meio do não julgamento de suas próprias atitudes ou atitudes do outro, aumentando a capacidade de perdão e se tornando mais abertos e menos defensivos. Ainda, a prática de atenção plena é eficaz em relação às características positivas do indivíduo, que se manifesta nos pensamentos, sentimentos e comportamentos, permitindo que esses indivíduos prosperem e consigam se adaptar às adversidades (Pizarro-Ruiz, Camblor, Del-Líbano, \& Llamazares, 2021).

Sendo assim, fica evidente que intervenções de atenção plena em períodos críticos podem vir a resultar em menores níveis em estresse e ansiedade. Levando em consideração o isolamento social, significa não apenas uma melhora no momento atual, mas também uma prevenção contra consequências futuras, que podem levar à quadros depressivos e ansiosos (PizarroRuiz, et al., 2021).

Ainda, segundo os autores, os indivíduos com maiores níveis de atenção plena conseguiram apontar os fatores positivos decorrentes das mudanças acerca da pandemia do covid-19 e desenvolver estratégias de enfrentamento adaptativas (Götmann \& Bechtoldt, 2021). A atenção plena pode representar um fator de proteção totalmente estável da intensidade do sofrimento mental dos indivíduos, quando praticado de forma frequente, pois, os efeitos de mindfulness corresponde a uma diminuição significativa do desconforto mental. $\mathrm{O}$ treinamento da meditação guiada influenciou positivamente a função da memória, resiliência cognitiva, na saúde mental, no equilíbrio e na diminuição de altos níveis de estresse, depressão e síndrome de burnout (Conversano, et al., 2020; Zhang, et al., 2021).Os autores ressaltam que o estado de atenção plena ajuda a lidar com as situações ansiogênicas, diminuindo a probabilidade de futuros problemas psicopatológicos e transtornos mentais crônicos, considerados traumáticos da recente pandemia (Conversano, et al., 2020).

A atenção plena, através da meditação guiada, tem resultados satisfatórios na intervenção de transtornos ligados a traumas, estresse e ansiedade. Cinco minutos por dia, rotineiramente, gera impactos positivos a longo prazo, melhorando o foco e a atenção (Zhu, Wekerle, Lanius, \& Frewen, 2019). Sua prática constante auxilia no desenvolvimento das funções cognitivas do cérebro, gerando impactos positivos em alguns casos clínicos como a ansiedade, depressão, dores crônicas, fibromialgia, entre outras. Além disso, o estado de atenção plena desencadeia sensações de otimismo, autoestima, criatividade, desenvolve habilidades interpessoais e espirituais, pois a meditação guiada tem relação com emoções positivas que geram bem-estar (Bezerra, 2018).

Nesse sentido, tendo em vista o distanciamento social ocasionado pela pandemia do covid-19 e o consequente aumento do uso de meios de comunicação através da internet, os estudos apresentados (El Morr, et al., 2020; Matiz, et al., 
2020; Pizarro-Ruiz, et al., 2021; Waller, Mistry, Jetly, \& Frewen, 2021; Yuan, 2021; Zhang, et al., 2021) demonstram os resultados efetivos em programas e práticas de mindfulness através da internet, possibilitando, dessa forma, a expansão e disseminação da prática de atenção plena à demais grupos sociais presente nas comunidades virtuais. Ainda, fatores como o baixo custo em intervenções online e maior alcance de pessoas, bem como a promoção de relações interpessoais, permite a acessibilidade que ultrapassa os limites impostos pelo espaço geográfico, e até mesmo, pelo tempo (El Morr, et al., 2020).

\subsubsection{Mindfulness e Resiliência na Pandemia do Covid-19}

A China foi o primeiro epicentro de infecção por covid-19, e por isso, o país precisou criar planos e táticas que contivesse o avanço do vírus, e suas estratégias serviram de modelo para os países que mais tarde se esbarrariam com o covid19. Com uma pandemia em curso, a população mundial precisou utilizar todas as suas técnicas de enfrentamento emocional, sendo a resiliência um importante constructo a ser destacado. Por conseguinte, a resiliência pode ser conceitualizada como a habilidade de enfrentar e recuperar-se frente as adversidades ao longo da vida (Taboada, Legal, \& Machado, 2006).

Além da China, outro país que se destacou no início da pandemia foi a Itália, Matiz e colaboradores (2020) desenvolveram um programa baseado em mindfulness com professores na Itália, antes e durante o início do bloqueio nacional do país para conter o avanço das contaminações por covid-19. Os participantes foram divididos em dois grupos de acordo o perfil nos níveis de resiliência, por isso, como os níveis de resiliência são distintos, os resultados obtidos também foram discrepantes, contudo, ambos os grupos apresentaram avanços positivos nos escores de atenção plena, empatia, traços de caráter, relações interpessoais positivas, interocepção e exaustão emocional. Ainda, no grupo com o menor nível de resiliência, percebeu-se melhora no bem-estar psicológico, como nos níveis de ansiedade, depressão e estresse. Os efeitos do estudo verificam que os indivíduos com baixa resiliência demonstraram resultados efetivos em relação aos participantes com alta resiliência, nota-se, então, que a discrepância nos dados pode ser devido ao perfil emocionalmente funcional dos participantes com maior resiliência e/ou fatores nos níveis de motivação, uma vez que os indivíduos que necessitam mais aliviar o seu sofrimento psicoemocional podem estar mais motivados a realizar os exercícios de mindfulness. A resiliência, dessa forma, contribui para o manejo de situações estressantes e na prevenção de problemas psicoemocionais, sendo essa capacidade passível de ser aprendida e empregada durante a vida através, inclusive, de exercícios de mindfulness.

Corroborando com os dados apresentados por Matiz e colaboradores, Yuan (2021) demonstra que as técnicas de mindfulness podem aumentar os níveis de resiliência e inteligência emocional também em adolescentes. Sendo assim, verificase a correlação entre esses dois constructos, resiliência e atenção plena, ambas habilidades que podem ser aprendidas e introduzidas no repertório de respostas de enfrentamento frente a eventos adversos que ocorrem ao longo da vida.

Contextualizando com o atual cenário de pandemia, desenvolver tais habilidades contribui para uma maior abertura para aceitar a realidade e as consequências oriundas desse contexto, proporcionando um olhar crítico, adaptativo e funcional frente a realidade mundial vivenciada, possibilitando que a população encare e valide suas emoções, sentimentos e construa estratégias a serem utilizadas não apenas no momento presente, como também, futuramente.

\subsection{Mindfulness: uma Estratégia de Intervenção na Depressão e Ansiedade}

Conforme apresentado anteriormente, o termo mindfulness pode ser desmembrado enquanto constructo teórico, enquanto exercícios informais e/ou formais de meditação e enquanto mecanismos mentais que podem ser desenvolvidos e/ou acionados através da prática dos exercícios. Na psicologia ocidental, cada vez mais têm se utilizado os exercícios de mindfulness enquanto estratégia interventiva em psicoterapia, ainda, percebe-se uma maior aproximação dessa prática com as psicoterapias cognitivo-comportamentais. 
Nesse sentido, os achados recentes na literatura sugerem a aplicação do programa de 8 semanas de Terapia Cognitiva Baseada em Mindfulness (MBCT) (Teasdale, Williams, \& Segal, 2016), associando os exercícios de mindfulness com estratégias cognitivas e técnicas comportamentais durante o processo psicoterapêutico (Almeida, et al., 2020; Bezerra, 2018; Pereira, Soares, \& Donadon, 2020).

Os resultados foram obtidos através da análise de estudos de casos clínicos: um estudo compreende onze estudos de casos de pacientes com sintomas de depressão (Almeida, et al., 2020); um estudo aborda o estudo de caso de uma paciente com sintomas de Transtorno do Pânico (Pereira, et al., 2020) e; um estudo aborda o estudo de caso de uma paciente com Síndrome de Burnout e sintomas de ansiedade moderada (Bezerra, 2018). A descrição das etapas do programa será listada abaixo, de acordo as características em comum entre os estudos.

A intervenção em mindfulness inicia-se com a utilização da psicoeducação, ou seja, psicoeduca-se o paciente sobre os princípios de mindfulness, como praticá-lo, suas vantagens, e, a importância da manutenção dos exercícios, em práticas formais e informais, para que os benefícios sejam duradouros e eficazes. Durante as sessões são aplicadas as técnicas, a cada semana é realizada uma técnica formal no setting terapêutico e sugerido ao paciente que ao longo da semana, ele pratique as técnicas informais. As técnicas formais, que são estruturadas em um limite de tempo, como as práticas de respiração e escaneamento corporal, podem ser narradas durante as sessões pelo psicoterapeuta e/ou guiadas através de gravações de áudio. Alguns exemplares de livros, como o livro Atenção Plena, dos autores Mark Williams e Danny Penman (2015) e o Manual Prático de Mindfulness, de Teasdale, Williams e Segal (2016), acompanham CD com faixas de áudio narrando as técnicas. Já as técnicas informais, são práticas que podem ser introduzidas no cotidiano do paciente e não tem uma estrutura em um limite de tempo, elas podem ser experienciadas através das sensações do sujeito, das suas vivências, buscando interromper o piloto automático e trazer a sua atenção, gentilmente, para o aqui e agora (Almeida, et al., 2020; Bezerra, 2018; Pereira, et al., 2020).

Entre as técnicas formais utilizadas ao longo das sessões, as mais citadas são: a) exercícios de respiração consciente, realizadas na posição sentada; b) exercícios de respiração consciente no espaço de três minutos; c) exercícios body scan (escaneamento corporal) e; d) exercícios de alongamento e respiração (alongamento consciente). Já as técnicas informais, estão pautadas no desenvolvimento da habilidade de trazer a consciência para atividades rotineiras do dia a dia, estando consciente e atento as refeições, ao escovar os dentes, ao tomar banho, ao fazer caminhadas e, também, perceber e estar consciente dos pensamentos, emoções e sensações que o sujeito venha a apresentar, possibilitando que o praticante observe suas vivências internas em uma perspectiva acolhedora, não julgadora, compassiva e com abertura para a experiência (Almeida, et al., 2020; Bezerra, 2018; Pereira, et al., 2020).

Associadas às práticas de mindfulness, também estão presentes ao longo das semanas as estratégias cognitivas que visam a regulação emocional, através da psicoeducação das emoções e sentimentos, o monitoramento dos pensamentos, ensinando o paciente que os seus pensamentos são eventos mentais, e não fatos concretos e, ainda, auxilia o paciente no desenvolvimento de comportamentos saudáveis e funcionais (Almeida, et al., 2020; Bezerra, 2018; Pereira, et al., 2020).

Um aspecto importante presente ao longo das sessões é o feedback, é importante o paciente eliciar um feedback a respeito de como está sendo a sua experiência com as práticas de mindfulness, quais as suas dificuldades, quais as técnicas que mais se identificou, para que o psicoterapeuta consiga auxiliar o paciente no decorrer do processo (Almeida, et al., 2020; Bezerra, 2018; Pereira, et al., 2020).

Na última semana do programa de mindfulness, ao final das sessões, é o momento onde o paciente poderá identificar quais foram os benefícios, mudanças e resultados ocorridos durante todo o processo e como permanecer fazendo uso das técnicas e exercícios após o termino dos encontros de psicoterapia (Almeida, et al., 2020; Bezerra, 2018; Pereira, et al., 2020).

É importante salientar que durante o processo de intervenção, ambos os autores utilizaram escalas e inventários a fim de mensurar e acompanhar os níveis dos sintomas apresentados pelos pacientes, como a Escala de Depressão, Ansiedade e 
Estresse (DASS-21), Escala de Ansiedade de Beck (BAI) e Inventário de Burnout de Maslach (MBI). Também, utilizou-se a Escala de Consciência e Atenção Mindfulness (MAAS), com o objetivo de mensurar os níveis de atenção plena (Almeida, et al., 2020; Bezerra, 2018; Pereira, et al., 2020).

Por fim, os resultados do programa de 8 semanas de Terapia Cognitiva Baseada em Mindfulness (MBCT), em ambos os estudos, foram positivos: a) os onze pacientes do estudo de Almeida e colaboradores (2020) obtiveram redução dos sintomas depressivos e melhora nos níveis de estresse e ansiedade. Os seis pacientes com maior remissão dos sintomas foram também os pacientes que mais realizaram os exercícios e práticas para além da sessão de psicoterapia; b) no estudo de Pereira e colaboradores (2020), a intervenção em mindfulness contribuiu para o desenvolvimento da atenção e concentração da paciente, aceitação, autopercepção e empatia, auxiliou na diminuição da ruminação, do reconhecimento dos pensamentos catastróficos, compreendendo-os enquanto eventos mentais e não necessariamente como eventos reais. Ainda, os exercícios de mindfulness auxiliaram na regulação emocional, aumento da tolerância, resolução de problemas e compaixão frente as limitações dos outros; c) por fim, no estudo de Bezerra (2018), os resultados demonstraram diminuição nos níveis de estresse e ansiedade, com remissão total dos sintomas psicossomáticos e contribuiu com o processo de autoconhecimento e regulação emocional da paciente.

O modelo de intervenção em mindfulness apresentado acima se refere a sua aplicação em psicoterapia, e diante dos resultados, percebe-se os benefícios que essa prática é capaz de proporcionar à saúde mental dos pacientes, associado ao processo psicoterapêutico. Contudo, o conhecimento dos princípios de mindfulness e o desenvolvimento dessa habilidade, é transversal a orientação direta de um psicólogo durante a psicoterapia, pois a disseminação da prática da atenção plena pode se manifestar de outras formas, através da literatura, recursos audiovisuais e cursos online, por exemplo, onde o profissional em psicologia e/ou instrutor de mindfulness, podem tornar essa prática acessível e contribuir com a saúde mental da população.

\section{Considerações Finais}

Com a pandemia do covid-19, a população mundial precisou se adaptar a novas formas de viver em sociedade. A longo prazo, são incertas as predições a respeito da saúde física da população, contudo, os prejuízos na saúde mental já são perceptíveis e ascendentes, em graus alarmantes. Diante desse contexto, se faz necessário o desenvolvimento de estratégias e repostas de enfrentamento saudáveis. Nesse sentido, a literatura utilizada para compor esse trabalho demonstrou a importância, a emergência e a multidimensionalidade de ações voltadas para a melhoria da saúde mental no que tange ao contexto da pandemia do covid-19, sendo a prática de mindfulness uma ferramenta pertinente e aplicável, mostrando resultados significativos na saúde individual e coletiva. No entanto, percebe-se que é crucial que mais ênfase seja atribuída a essa problemática, se manifestando através de pesquisas e estudos futuros, que venham a trazer mais evidências sobre o assunto, agregando significativo valor à saúde mental da população.

\section{Referências}

Almeida, N. de O., Demarzo, M., \& Neufeld, C. B. (2020). Terapia cognitiva baseada em mindfulness no atendimento clínico individual de depressão. SMAD. Revista eletrônica saúde mental álcool e drogas, 16(3), 55-63. 10.11606/issn.1806-6976.smad.2020.165509.

American Psychiatric Association. (2014). Manual diagnóstico e estatístico de transtornos mentais: DSM-5. (5a ed.), M. I. C. Nascimento, Trad.). Porto Alegre, RS: Artmed.

Antonova, E., Amaratunga, K., Wright, B., Ettinger, U., \& Kumari, V. (2016) Schizotypy and mindfulness: magical thinking without suspiciousness characterizes mindfulness meditators. Schizophr Res Cogn. May; 5, 1-6. 10.1016/j.scog.2016.05.001.

Barros, M. B. A., Lima, M. G., Malta, D. C., Szwarcwald, C. L., Azevedo, R. C. S. De, Romero, D. et al. (2020) Relato de tristeza/depressão, nervosismo/ansiedade e problemas de sono na população adulta brasileira durante a pandemia de covid-19. Epidemiol. Serv. Saúde, Brasília, 29 (4), 2020. 10.1590/S1679-49742020000400018. 
Bezerra, A. C. V., Silva, C. E. M. Da, Soares, F. R. G., \& Silva, J. A. M. da. (2020) Fatores associados ao comportamento da população durante o isolamento social na pandemia de covid-19. Ciênc. saúde coletiva, Rio de Janeiro, 25 (1), 2411-2421. 10.1590/1413-81232020256.1.10792020.

Bezerra, A. D. (2018) A prática de Mindfulness na terapia cognitiva comportamental: um caso clínico (Trabalho de conclusão de curso)

Campina Grande, 01-27. http://dspace.sti.ufcg.edu.br:8080/jspui/bitstream/riufcg/14804/1/ALESSANDRO\%20DUTRA\%20BEZERRA\%20$\% 20$ TCC\%20PSICOLOGIA\%202018.pdf.

Bhuiyan, A. I., Sakib, N., Pakpour, A. H., Griffiths, M. D., \& Mamun, M. A. (2020) Covid-19-related suicides in bangladesh due to lockdown and economic factors: case study evidence from media reports. Int J Ment Health Addiction, 1-6. 10.1007/s11469-020-00307-y.

Ministério da Saúde. (2020) Ministério da Saúde divulga resultados preliminares de pesquisa sobre saúde mental na pandemia. https://antigo.saude.gov.br/noticias/agencia-saude/47527-ministerio-da-saude-divulga-resultados-preliminares-de-pesquisa-sobre-saude-mental-na-pandemia.

Conversano, C., Di Giuseppe, M., Miccoli, M., Ciacchini, R., Gemignani, A., \& Orrù, G. (2020) Atenção plena, idade e gênero como fatores de proteção contra sofrimento psicológico durante a pandemia de COVID-19. Frente. Psychol. 11, 1900. 10.3389/fpsyg.2020.01900.

El Morr, C., Ritvo, P., Ahmad, F., Moineddin, R., \& Equipe, M.V.C. (2020). Effectiveness of an 8-week web-based mindfulness virtual community intervention for university students on symptoms of stress, anxiety, and depression: randomized controlled trial. JMIR mental health, 7(7), 18595. 10,2196 / 18595.

Foroughi, A., Sadeghi, K., Parvizifard, A., Moghadam, A. P., Davarinejad, O., Farnia, V., \& Azar, G. (2020). A eficácia da terapia cognitiva baseada na atenção plena para reduzir a ruminação e melhorar a atenção plena e a autocompaixão em pacientes com depressão resistente ao tratamento. Trends in Psychiatry and Psychotherapy, 42 (2), 138-146. 10.1590/2237-6089-2019-0016.

De Frias, C. M., \& Whyne, E. (2015). Stress on health-related quality of life in older adults: the protective nature of mindfulness. Aging \& mental health, 19(3), 201-206. 10.1080/13607863.2014.924090.

Germer, C. K., Siegel, R. D., \& Fulton, P. R. Mindfulness e psicoterapia. (2a ed.; M. C. G. Monteiro, Trad.) Porto Alegre: Artmed, 2016.

Gonzalez-Diaz, J. M., Cano, J. F., \& Pereira-Sanchez, V. Psychosocial impact of covid-19-related quarantine: reflections after the first case of suicide in colombia. Cadernos de Saúde Pública. 36, 6. 10.1590/0102-311X00117420.

Götmann, A., \& Bechtoldt, M. N. (2021). Coping with covid-19 - longitudinal analysis of coping strategies and the role of trait mindfulness in mental wellbeing. Personality and individual differences, 175, 110695. 10.1016/j.paid.2021.110695.

Haliwa, I., Lee, J., Wilson, J., \& Shook, N. J. (2020) Mindfulness and engagement in covid-19 preventive behavior. Preventive Medicine Reports, $20,101246$. 10.1016/j.pmedr.2020.101246.

Hayes, S. S., Strosahl, K. D., \& Eilson, K. G. (1999) Acceptance and commitment therapy: an experiential approach to behavior change. (1 ${ }^{\text {a }}$ ed.) New York: Guilford Press.

Kabat-Zinn, J. (1990) Full catastrophe living: using the wisdom of your body and mind to face stress, pain and illness. Dell.

Lemos, I. S., Carvalho, J. V. S. de, Mendes, M. T. G., \& Brys, I. (2021). Mindfulness e relaxamento: os efeitos de um programa com funcionários de um hospital universitário. Estudos de Psicologia, 38, e 190128. 10.1590/1982-0275202138e190128.

Linehan, M. M. (1993) Cognitive-behavioral treatment of bordeline personality disorder. Guilford Press.

Maleki, M., Khorramnia, S., Foroughi, A., Amiri, S., \& Amiri, S. (2021) Comparing the effectiveness of the unified protocol in combination with an additional mindfulness treatment to the unified protocol alone as treatment for adolescents diagnosed with emotional disorders. Trends Psychiatry Psychother. 43 (1), 5764. 10.47626/2237-6089-2020-0046.

Matiz, A., Fabbro, F., Paschetto, A., Cantone, D., Roberto, A., \& Crescentini, C. (2020). Positive impact of mindfulness meditation on mental health of female teachers during the covid-19 outbreak in italy. International journal of environmental research and public health. 17(18), 6450. 10.3390/ijerph17186450.

Mertens, G., Gerritsen, L., Duijndam, S. N. C., Salemink, E., \& Engelhard, I. (2020). Fear of the coronavirus (covid-19): predictors in an online study conducted in march 2020. PsyArXiv Preprints. 10.31234/osf.io/2p57j.

Neff, K., \& Germer, C. (2019) Manual de mindfulness e autocompaixão: um guia para construir forças internas e prosperar na arte de ser seu melhor amigo. ( $1^{\text {a }}$ ed.; S. M. M. da Rosa, Trad.) Porto Alegre: Artmed.

Penman, D., \& Williams, M. (2015) Atenção Plena - mindfulness: como encontrar a paz em um mundo frenético. (1 ${ }^{\text {a }}$ ed.; I. Korytowski, Trad.) Rio de Janeiro: Sextante editora.

Pereira, C. C. B., Soares, T. A. B., \& Donadon, M. F. (2020) Mindfulness: uma ferramenta complementar na manutenção e remissão dos sintomas de transtorno de pânico. Revista eixo. 9, 3. http://revistaeixo.ifb.edu.br/index.php/RevistaEixo/article/view/770/535.

Pizarro-Ruiz, J. P., Camblor, N. O., Del-Líbano, M., \& Llamazares, M. C. E. (2021). Influence on forgiveness, character strengths and satisfaction with life of a short mindfulness intervention via a spanish smartphone application. International journal of environmental research and public health, $18(2)$, 802. 10.3390/ijerph18020802.

Rodriguez-Vega, B., Palao, Á., Muñoz-Sanjose, A., Torrijos, M., Aguirre, P., Fernández, A. et al. (2020). Implementation of a mindfulness-based crisis intervention for frontline healthcare workers during the covid-19 outbreak in a public general hospital in madrid, spain. Frontiers in psychiatry, 11,562578 . $10.3389 /$ fpsyt.2020.562578.

Saricali, M., Satici, S. A., Satici, B., Gocet-Tekin, E., \& Griffiths, M. D. (2020). Fear of covid-19, mindfulness, humor, and hopelessness: a multiple mediation Analysis. International journal of mental health and addiction, 1-14. 10.1007/s11469-020-00419-5. 
Research, Society and Development, v. 10, n. 15, e545101523559, 2021

(CC BY 4.0) | ISSN 2525-3409 | DOI: http://dx.doi.org/10.33448/rsd-v10i15.23559

Segal, Z. V., Williams, J. M. G., \& Teasdale, J. D. (2018) Mindfulness-based cognitive therapy for depression (2 ${ }^{\mathrm{a}}$ ed.) New York: Guilford Press.

Taboada, N. G., Legal, E. J., \& Machado, N. (2006). Resiliência: em busca de um conceito. Journal of Human Growth and Development, 16(3), 104-113. http://pepsic.bvsalud.org/scielo.php?script=sci_arttext\&pid=S0104-12822006000300012\&lng=pt\&tlng=pt.

Teasdale, J. D.; Williams, M., \& Segal, Z. (2016). Manual Prático de Mindfulness (Meditação da Atenção Plena). (1ª ed.; C.G, Duarte, Trad.) São Paulo: Pensamento.

Waller, M., Mistry, D., Jetly, R., \& Frewen, P. (2021). Meditating in Virtual Reality 3: 360 Video of Perceptual Presence of Instructor. Mindfulness, 1-14. 10.1007/s12671-021-01612-w.

World Health Organization. (2017). Depression and other common mental disorders - global health estimates. Geneva. http://apps.who.int/iris/bitstream/10665/254610/1/WHO-MSD-MER-2017.2-eng.pdf?ua=.

Xiang, Y. T., Yang, Y., Li, W., Zhang, L., Zhang, Q., Cheung, T., \& Ng, C. H. (2020). Timely mental health care for the 2019 novel coronavirus outbreak is urgently needed. The lancet. Psychiatry, 7(3), 228-229. 10.1016/S2215-0366(20)30046-8.

Yuan Y. (2021). Mindfulness training on the resilience of adolescents under the covid-19 epidemic: a latent growth curve analysis. Personality and individual differences, 172, 110560. 10.1016/j.paid.2020.110560.

Zhang, H., Zhang, A., Liu, C., Xiao, J., \& Wang, K. (2021). A brief online mindfulness-based group intervention for psychological distress among chinese residents during covid-19: a pilot randomized controlled trial. Mindfulness, 1-11. 10.1007/s12671-021-01618-4.

Zhu, J., Wekerle, C., Lanius, R., \& Frewen, P. (2019). Trauma- and stressor-related history and symptoms predict distress experienced during a brief mindfulness meditation sitting: moving toward trauma-informed care in mindfulness-based therapy. Mindfulness. 10, 1985-1996. 10.1007/s12671-019-01173z. 